\title{
Development of automatic matching and image quality improving methods for scattering 3D display
}

\section{AUTHOR(S):}

Ohashi, Yoshiki; Ishii, Hirotake; Shimoda, Hiroshi; Endo, Ryuta; Fujii, Takuya

\section{CITATION:}

Ohashi, Yoshiki ...[et al]. Development of automatic matching and image quality improving methods for scattering 3D display. 2017 IEEE International Conference on Systems, Man, and Cybernetics (SMC) 2017

\section{ISSUE DATE:}

2017-10

URL:

http://hdl.handle.net/2433/236472

\section{RIGHT:}

(c) 2017 IEEE. Personal use of this material is permitted. Permission from IEEE must be obtained for all other uses, in any current or future media, including reprinting/republishing this material for advertising or promotional purposes, creating new collective works, for resale or redistribution to servers or lists, or reuse of any copyrighted component of this work in other works.; この論文は出版社版でありません。引用の際には出版社版をご確認ご利用ください。; This is not the published version. Please cite only the published version. 


\section{Development of Automatic Matching and Image Quality Improving Methods for Scattering 3D Display}

\author{
Yoshiki Ohashi, Hirotake Ishii \\ and Hiroshi Shimoda \\ Graduate School of Energy Science, \\ Kyoto University \\ Kyoto, Japan 606-8501 \\ Email: ohashi@ei.energy.kyoto-u.ac.jp
}

\author{
Ryuta Endo \\ Present : FreakOut Holdings, inc. \\ Tokyo, Japan 106-0032
}

\author{
Takuya Fujii \\ Present : SoftBank Corp. \\ Tokyo, Japan 105-7309
}

\begin{abstract}
Up to this day, many types of 3D displays have been developed. However, there remain some challenges in the displays, for example, restriction of view points and vergenceaccomodation conflict. A 3D display was proposed in the past using light diffusion within 3-dimensionally positioned micro voids processed in a crystal glass cuboid. It is able to present 3D objects to any number of simultaneous viewers at any viewpoint, without wearing any kind of special glass-like equipments. However, in order to successfully present 3D objects, the projection must be adjusted precisely for each individual micro void by investigating the correspondence between each projector pixel and micro voids. This process not only requires a great amount of user's effort but also consumes a lot of time. Hence, for this research, pixel-to-void matching methods which can automatically find out correspondence between each projector pixel and micro voids by using a camera were developed. These methods were verified in the experimental systems to be able to perform such matching procedure automatically and accurately. In addition, using multiple projectors, brightness and resolution of the display was improved. Two types of the displays were developed, one aimed to improve especially its brightness and the other aimed to improve especially its resolution. While the latter one resulted in higher resolution but in subjective evaluation experiment no improvement of visibilities was verified, the former one resulted in higher brightness and it was verified to have improved visibilities in subjective evaluation experiment.
\end{abstract}

Keywords-volumetric display; optical scatterers; glasses-free

\section{INTRODUCTION}

In recent years, 3D displays became similar to our lives. 3D movies and 3D television are good examples. However, most of these 3D displays have problems, such as limitation of simultaneous viewers, restricted viewing zone and vergence-accommodation conflicts[1]. Therefore, new types of 3D displays have been studied and developed to solve the problems mentioned above. Especially, 3D displays which can be observed without special equipments, such as 3D glasses are actively being studied.

Iwasawa et al. developed REI, an automultiscopic projection display[2]. This display can provide high resolution image respectively for each view point by using a projector array which includes as many projectors as view points and superimposing projecting images on to the screen consisting of anisotropic diffuser and condenser lens. Furthermore, even though this is a flat panel 3D display, it will not incur vergenceaccommodation conflicts because its designed distance between view points is shorter than pupil diameters. However, it has some problems. According to the increase of view points, the device becomes larger, more complex and more costly. Viewing angle is limited near the front of the screen. The distance to the screen which viewers can perceive proper stereoscopic effects is restricted, and so on.

The displays that display images in volumetric regions were also developed, such as Interactive $360^{\circ}$ Light Field Display by Jones et al.[3] and Aerial 3D Display by Ishikawa et al.[4]. These displays can be observed by the naked eye, do not incur vergence-accommodation conflicts and have no limitation for viewable directions and distance. Interactive $360^{\circ}$ Light Field

Display projects images on to the mirror rotating in high speed. Varying the projection image according to the angle of the mirror, it can provide different images corresponding to the viewing position. It has high resolution but some problems. For example, the displayed image flickers and it is hard to enlarge the device. Aerial 3D Display displays 3D images in mid-air by plasma produced by high power laser. Though it can display images in mid-air, it is difficult to display smooth movie because of its low resolution and slow refresh rate. In addition, it also has the disadvantage that it cannot display full-color image.

Besides them, Nayar et al. developed 3D display using light diffusion within 3-dimensionally positioned micro regions (scattering display)[5]. Scattering display's body is glass cuboid in which a lot of micro voids are processed. It displays 3D image by using projector to lighten each voids. The void lit by projector scatters the light and works as a voxel thus displaying images in volumetric regions as well. It also has the features that it can be observed by naked eyes, it does not incur vergence-accommodation conflicts and it has no limitation for viewable directions and distance. To display 3D 
image by scattering display, it is needed to investigate which pixel of projector lighten which void in the display's body and register projector's pixels with corresponding voids. Nayar et al. performed this matching almost manually and it took very long time and much manpower. The more voids processed in display's body, the longer it takes to correspond pixels with voids and the higher resolution(density of the voids) the display has, the higher accuracy of matching is required, which makes manual matching difficult. This is one of the obstructions to enhancing scattering display's resolution. On the other hand, while Nayar et al. used only one projector to lighten voids, it is expected that brightness and resolution of the displayed image can be improved by multiple projectors.

Hence, in this study, partly-automatic matching algorithm of scattering display was developed. Thanks to this, rematching when positional shift occurs became easier than before. In addition, it enabled more accurate matching even for the display which has dense voids, and the resolution of the display was increased. Moreover, we tried to improve brightness, contrast ratio and resolution of displayed image by using 4 projectors, and verified the effect of that.

\section{Principle of the 3D Display and Problems}

The principle of scattering display is shown in Fig.1. A lot of micro voids are processed 3-dimensionally in the glass cuboid shown in the center of the figure. Arbitrary 3D images can be displayed by using projectors to lighten voids and controlling the luminescence of individual voids. When we do so, the information about correspondences between the projector's pixel and the void lit by that projector's pixel is needed. In this study, we call the procedure to obtain that information "pixel-to-void matching" or simply "matching". Nayar et al. manually performed matching for a small number of voids and used the correspondence information of them to obtain the function expressing the correspondence of the rest voids and the projector's pixels which lighten those voids. However, the correspondence information acquired from the function included incorrect correspondence, and they manually corrected them 1 by 1 , it took quite a long time and much manpower. Required time for matching becomes longer according to the number of voids, and higher accuracy is required according to the density of voids, which makes manual matching difficult.

In addition to this, if multiple voids are aligned on the locus of 1 laser light from projector, such voids are hard to be used for displaying because they cannot be controlled their luminescence individually. Hence, too dense voids result in decrease of voids which can be lighten individually, instead of increasing the resolution.

And furthermore, there is a trade-off between the number of voids which can be positioned without overlapping with other voids on the locus of the same laser light and size of each void(the bigger the void is, the brighter it can be luminous because it can be lit by more pixels' laser light of the projector).

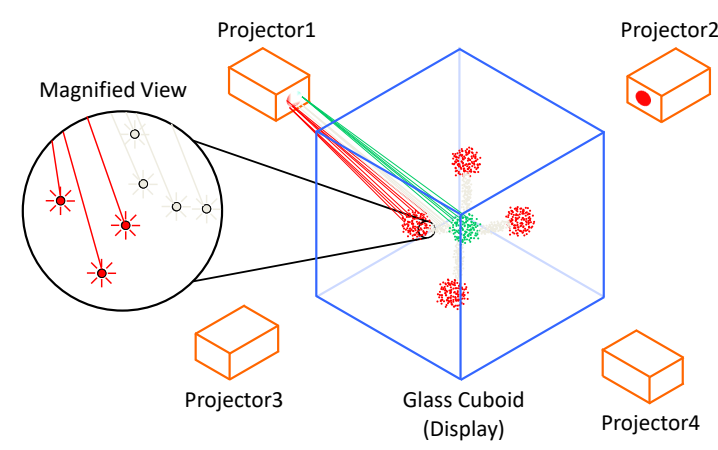

Fig. 1. Principle of scattering display.

\section{PROPOSALS FOR IMPROVEMENT}

In this section, the way to solve the problems mentioned in section 2 is proposed.

\section{A. Automization of the Pixel-to-Void Matching}

To display 3D images by scattering display, it is necessary to precisely control laser light output from the projector and luminescence of individual voids. If this controlling is performed by calculating the correspondence between pixels and voids based only on the design informations of voids' alignments and the relative position and pose of the projector to the glass body, it fails to lighten the target voids because of the processing errors of voids and the assembling error of the projector's position. Therefore, pixel-to-void matching has to be performed after assembling the device. In this study, automatic matching method was proposed. Proposed method uses a camera to detect the luminescence of voids emitted when they are lit by the laser light from projector. The 3D position of detected void can be calculated from the pixel coordinate of the projector used for lightening, pixel coordinate of the void in captured image and the relative position and pose to the display body of both projector and camera which acquired in advance.

There are various laser patterns which can be used to lighten voids for matching, the method investigating the correspondence between pixels and voids by lightening 1 pixel by 1 pixel and detecting the void lit by that 1 pixel is expected to be able to perform matching most accurately. We named this "Pixel-Scan method". However, while Pixel-Scan method is expected to be accurate, it needs as many times of capturing as the number of pixels the projector has. To solve this problem, it is effective to extract pixels which are likely to lighten at least 1 void and to perform matching only of those extracted pixels. To extract such pixels, we used shadows made by lit voids behind the display's body. As shown in Fig.2, inputting fully white image into a projector and irradiating the display's body by all laser light output from the projector, shadows of voids are made behind the display's body. The projector's pixels corresponding to the shadow are the very pixels likely to lighten voids. We named the way to perform matching with this pixel extracting technique "Filtered-Pixel-Scan method".

Other two fast matching methods which reduce the number 


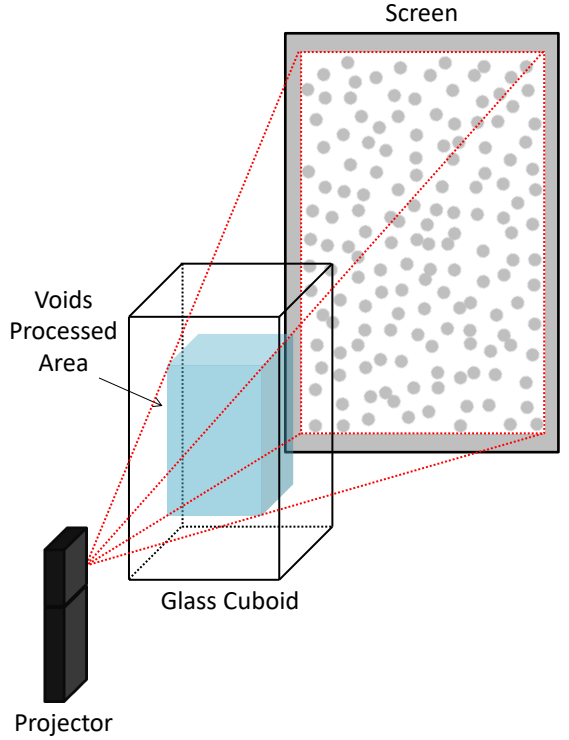

Fig. 2. Voids' shadows behind the display's body.

of capturing by using designed laser patterns were developed, namely; "Line-Scan method" and "Structured-Light method". Line-Scan method uses a vertical or a horizontal line laser as shown in Fig.3. Logical AND image of two images captured while irradiating a vertical and a horizontal line laser represents the state of voids while irradiating laser of 1 pixel located on the intersection point of vertical and horizontal lines. To use these virtually generated images, matching can be performed with fewer number of capturing which equals to the sum of the number of columns and rows of projector's image. StructuredLight method irradiates fringe patterns' laser along to vertical or horizontal line. First, the width of each fringe is set to the half number of the columns or rows of projector's image and capturing the state of voids while irradiating this fringe image and inversed fringe image. The fringe width is shortened to half after capturing, and repeat capturing and shortening till fringe width becomes 1 pixel. The equivalent images to the images captured while irradiating any 1 pixel laser light can be generated by taking the logical AND of some images captured while irradiating various fringe patterns' laser. StructuredLight method needs fewer number of capturing than Line-Scan method does (logarithmic order for projector's resolution).

\section{B. Multiple Projectors for Higher Resolution and Brightness}

While Nayar et al. used only one projector to lighten voids, it is expected that brightness and resolution of the displayed image can be improved by multiple projectors. First, the principle to improve the brightness by multiple projectors is shown in Fig.4. As shown in the figure, irradiating laser lights from multiple projectors to the same void, the brightness of that void is improved. Next, the principle to improve the resolution by multiple projectors is shown in Fig.5. As shown in the figure, if two or more voids are aligned on the locus of 1 laser light from a projector, the projector cannot control their luminescence individually, but lightening from another angle

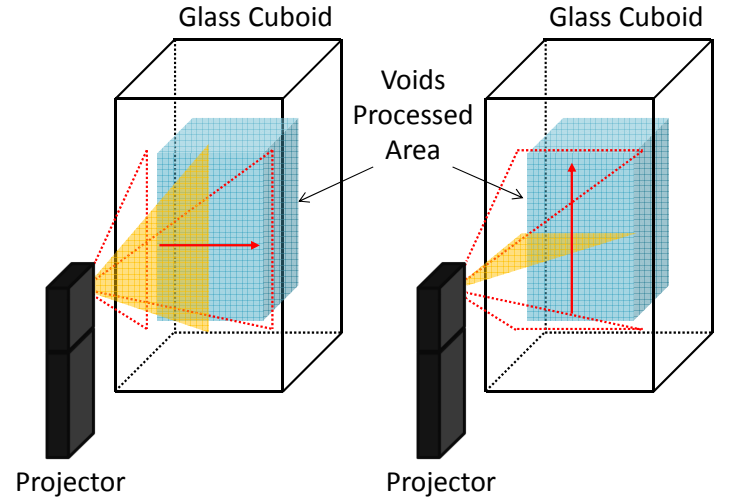

(a)Project along vertical line

(b)Project along horizontal line

Fig. 3. Laser projection in line-scan method matching.

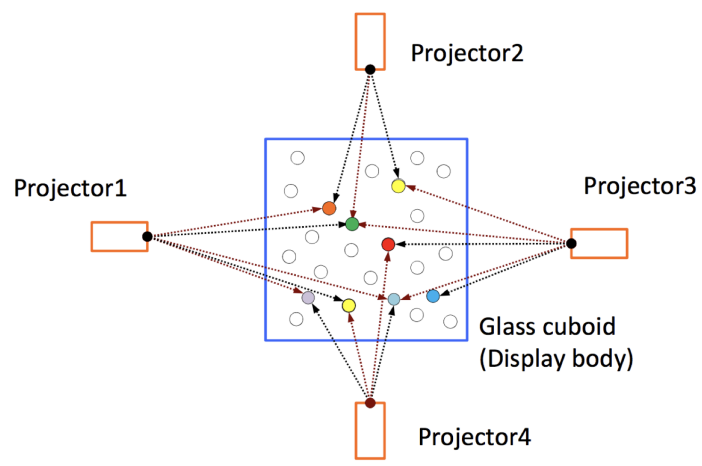

Fig. 4. Principle to improve the brightness by multiple projectors.

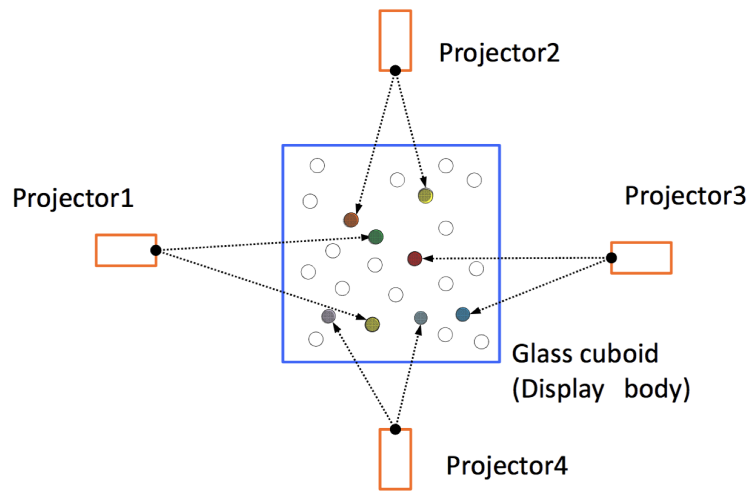

Fig. 5. Principle to improve the resolution by multiple projectors.

by using another projector, their luminescence can likely be controlled individually. The void can be used for displaying if at least 1 projector can control its luminescence individually. So, using multiple projectors to lighten voids, the number of the voids which can be effectively used increases, and consistently, the resolution of the display improves. In these ways, the quality of the displayed image can be enhanced by multiple projectors. 
TABLE I

DESIGNS OF THREE Displays

\begin{tabular}{|c|c|c|c|}
\hline & $\begin{array}{c}\text { 1-Projector } \\
\text { Display }\end{array}$ & $\begin{array}{c}\text { High-Brightness } \\
\text { Display }\end{array}$ & $\begin{array}{c}\text { High-Resolution } \\
\text { Display }\end{array}$ \\
\hline $\begin{array}{c}\text { Number of } \\
\text { Projector(s) }\end{array}$ & 1 & 4 & 4 \\
\hline $\begin{array}{c}\text { Number of } \\
\text { Camera(s) }\end{array}$ & 1 & 2 & 1 \\
\hline $\begin{array}{c}\text { Number of } \\
\text { Voids }\end{array}$ & 3000 & 3000 & 10,600 \\
\hline $\begin{array}{c}\text { Diameter of } \\
\text { Each Void }\end{array}$ & $0.2 \mathrm{~mm}$ & $1.0 \mathrm{~mm}$ & $0.2 \mathrm{~mm}$ \\
\hline
\end{tabular}

\section{DEMONSTRATION IN EXPERIMENTAL DisPlay DEVICE}

In this section, two points were verified by experimental display devices. One is whether automatic matching works well or not, the other is whether the quality of the image can be improved by multiple projectors or not. First, we developed the scattering display with only 1 projector (hereafter, 1-projector display) and confirmed that automatic matching algorithms worked well. Second, we developed the two types of displays with 4 projectors, designed to improve especially brightness (hereafter, high-brightness display) and designed to improve especially resolution (hereafter, high-resolution display), and compared the displayed images respectively with 1-projector display to verify that multiple projectors improved image qualities. The designs of the three displays are shown in Fig.I. The detail information for each display is mentioned below.

\section{A. 1-Projector Display}

The schematic view and the appearance of 1-projector display and the specification of the glass cuboid are shown in Fig.6, Fig.7 and Fig.8. The device is composed of a glass cuboid as display's body, a projector to lighten voids, a camera for capturing the state of voids in matching procedure and a computer to operate the system. The glass cuboid is $\mathrm{H} 140 \times \mathrm{W} 80 \times \mathrm{D} 80 \mathrm{~mm}$, and 3000 voids was processed in the central $\mathrm{H} 80 \times \mathrm{W} 50 \times \mathrm{D} 50 \mathrm{~mm}$ region by laser induced damage[6]. The shape of each void was sphere and the diameter was $0.2 \mathrm{~mm}$. 16 voids(markers) other than 3000 voids were processed in the glass in order to estimate the position and pose of both projector and camera. The density of voids is 15 [voids $/ \mathrm{cm}^{2}$ ] which is more than 4 times higher than that of Nayar's (about 3.57[voids $\left./ \mathrm{cm}^{2}\right]$ ). Laser projector PicoPro (Celluon Inc.) was used because of its focus-free feature. While Nayar et al. used DLP for the light source and projected images orthographically by using Fresnel lens, we used only laser projector(s) to avoid the device becoming too complex. Considering the throw ratio of the projector, the distance between the projector and the glass cuboid was set to $120 \mathrm{~mm}$ to be able to cover the entire region in which voids were processed. The specifications of the projector and the camera is shown in TABLE II and TABLE III.

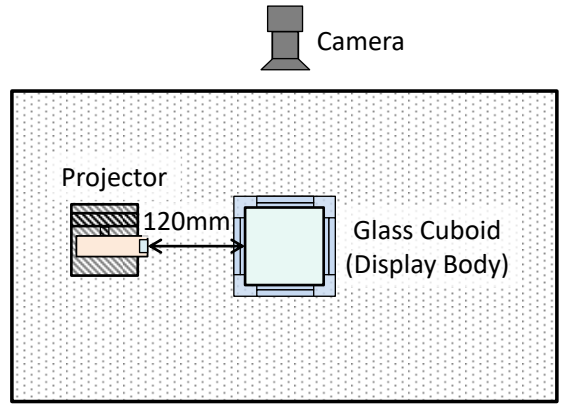

Fig. 6. Schematic view of 1-projector display.

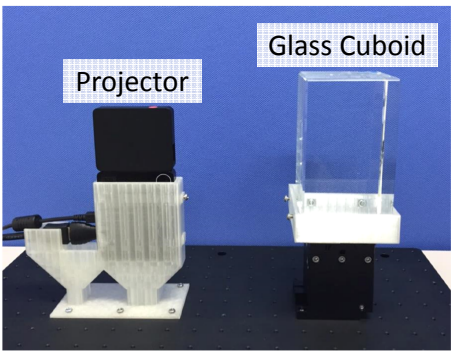

Fig. 7. Appearance of 1-projector display

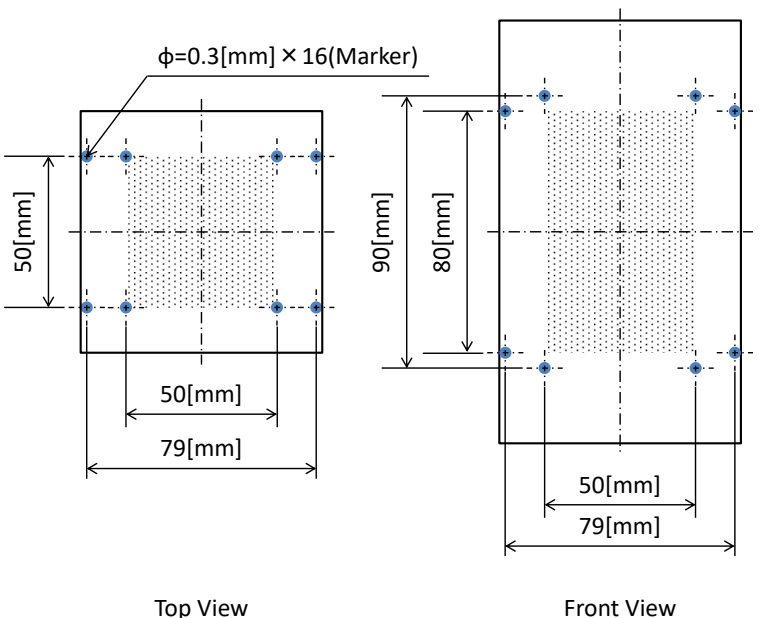

Fig. 8. Arrangement of voids in glass cuboid.

TABLE II

SPECIFICATION OF LASER Projector

\begin{tabular}{|c|c|}
\hline Product Name & PicoPro \\
\hline Display Method & Laser \\
\hline Resolution & $1920 \times 720$ [pixel] \\
\hline Brightness & 32 [ANSI Lumens] \\
\hline Contrast Ratio & $8000: 1$ \\
\hline Refresh Rate & $60[\mathrm{~Hz}]$ \\
\hline Color Depth & RGB $24[\mathrm{bit}]$ \\
\hline Throw Ratio & 1.3 \\
\hline Focus & Focus free (always in focus) \\
\hline
\end{tabular}

\section{B. High-Brightness Display}

The schematic view and the appearance of high-brightness display are shown in Fig.9 and Fig.10. The size of the glass 
TABLE III

SPECIFICATION OF CAMERA

\begin{tabular}{|c|c|}
\hline Model Number & FL3-U3-13S2C-CS \\
\hline Sensor Type & CMOS \\
\hline Sensor Format & $1 / 3 ”$ \\
\hline Resolution & $1280 \times 1024[$ pixel] \\
\hline Maximum Frame Rate & $120[\mathrm{fps}]$ \\
\hline Interface & USB3.0 \\
\hline Exposure & $8[\mu \mathrm{m}]-1[\mathrm{~s}]$ \\
\hline Lens Mount & CS-Mount \\
\hline Model Number of Mounted Lens & YV2.8x2.8SA-2 \\
\hline
\end{tabular}

Camera1

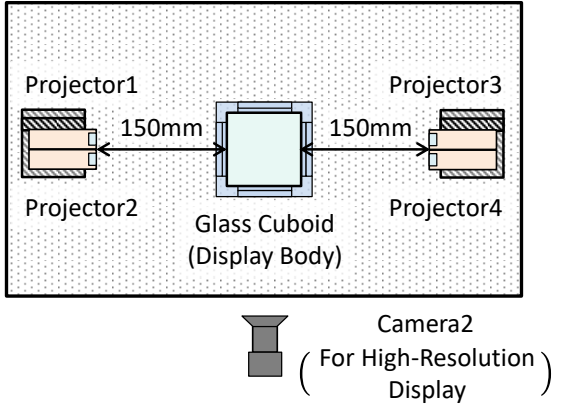

Fig. 9. Schematic view of high-brightness display and high-resolution display.

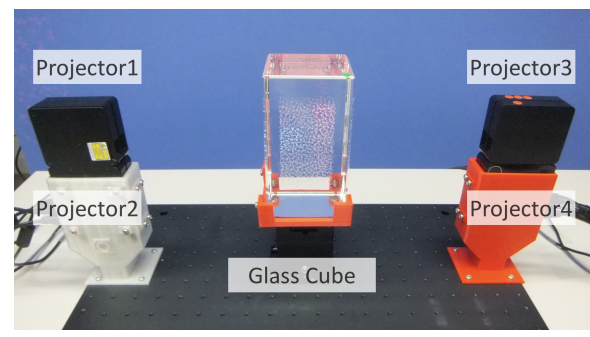

Fig. 10. Appearance of high-brightness display.

cuboid, the number and arrangement of voids, and the number and arrangement of markers are the same with 1-projector display's. The shape of each void was sphere and the diameter was $1.0 \mathrm{~mm}$. Considering the throw ratio of the projector, the distance between each projector and the glass cuboid was set to $150 \mathrm{~mm}$ to be able to cover the entire region in which voids were processed. Detection of voids' luminescence during matching procedure sometimes failed because the voids were enlarged and these enlarged voids sometimes obstruct the light emitted by other voids. To solve this, two cameras were used for covering the blind spots of each other. The specifications of all projectors and cameras were the same with those of 1-projector display's.

\section{High-Resolution Display}

The schematic view of high-resolution display is the same with that of high-brightness display's except for camera2 (shown in Fig.9). The appearance is shown in Fig.11. The size

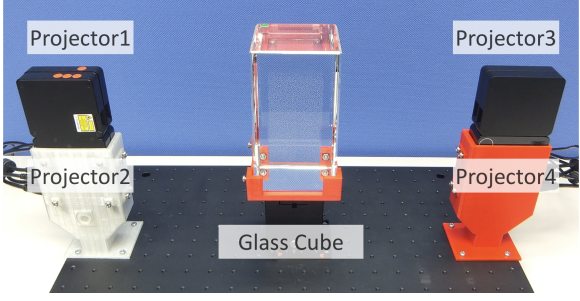

Fig. 11. Appearance of high-resolution display.

of the glass cuboid and the number and the arrangement of markers are the same with other 2 displays' but the number of voids was set to 10,600 to improve the resolution. The shape of each void was sphere and the diameter was $0.2 \mathrm{~mm}$. Considering the throw ratio of the projector, the distance between each projector and the glass cuboid was set to $150 \mathrm{~mm}$ to be able to cover the entire region in which voids were processed. The specifications of all projectors and the camera were the same with those of 1-projector display's.

\section{Results and Consideration}

First, Filtered-Pixel-Scan method matching was carried out with 1-projector display. Displayed images generated by using the result of the matching is shown in Fig.12. As shown in the figure, 3D shapes were displayed at the distinguishable level, so it can be said that automatic matching was success. Line-Scan method matching and Structured-Light method matching was also carried out with 1-projector display. Both images generated by using the result of Line-Scan method matching and Structured-Light method matching were distinguishable as well as images by Filtered-Pixel-Scan method matching but a slight degradation in quality of images was observed (There were some unnecessary luminescence out of $3 \mathrm{~d}$ shapes.). Therefore, in following experiments, FilteredPixel-Scan method was employed.

Second, the images displayed by 3 types of scattering displays were compared. The comparison between the images displayed by high-brightness display and 1-projector display is shown in Fig.13. All pictures in this figure were captured in the same light environment and by the same camera (with the same settings). Judging from this figure, it is obvious that the brightness of images was improved in high-brightness display. In addition, subjective evaluation experiment was conducted to verify whether the improvement of brightness leads to improvement of visibility. The experiment was conducted with 12 participants and participants were asked to answer the shape of the displayed images under various illuminations and with various background color. The results showed that correct answer rate of high-brightness display was higher than that of 1-projector display under the high illumination environment especially with brighter background color.

And then, the comparison between the images displayed by high-resolution display and 1-projector display is shown in Fig.14. It can be seen that high-resolution display has denser voids and the contours of the displayed shapes is clearer. Sub- 


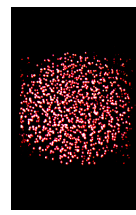

Sphere

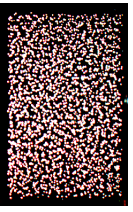

Cuboid

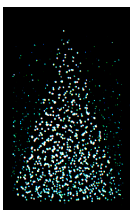

Cone

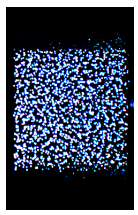

Cube
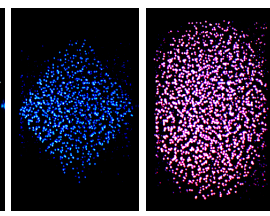

ctahedron Spheroid
Fig. 12. Displayed images by 1-projector display.

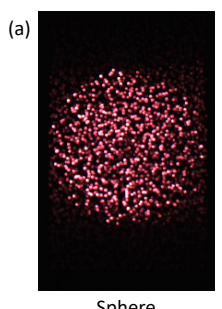

Sphere

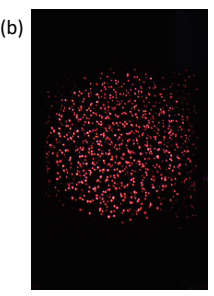

Sphere

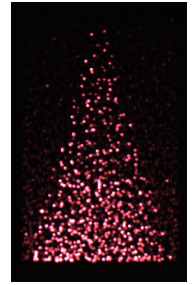

Cone

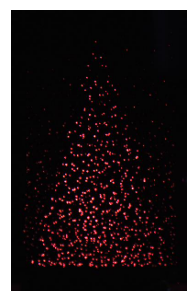

Cone
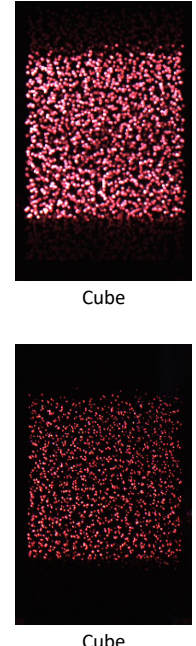

Fig. 13. Comparison between displayed images by (a)high-brightness display and (b)1-projector display.

jective evaluation experiment was conducted as well to verify whether the improvement of resolution leads the improvement of visibility or not. The experiment was conducted for 12 participants and participants were asked to answer the shape of the displayed images which gradually became larger and larger as soon as they understood what shape the image was. The results showed that there was no difference between highbrightness display and 1-projector display. One of the reasons why the score of high-brightness display did not improve is that there were unnecessary luminescence out of $3 \mathrm{~d}$ shapes. It was likely that dense voids led to increase of laser light which lighten a void strongly and simultaneously lighten other void at the levels undetectable to the camera, resulting in the increase of unnecessary luminescence. To prevent this, it is essential to enhance the accuracy of luminescence detection by camera during matching procedure. To enhance the accuracy, not only using camera with high-sensitivity, but also modifying the patterns of the laser output from the projector may be effective.

\section{CONCLUSiON}

In this study, we developed the automatic matching methods for scattering display. Developed methods enabled to perform matching without manpower even for the display with higher resolution, which made improvement of scattering display's resolution easier. Moreover, the method to enhance the im-

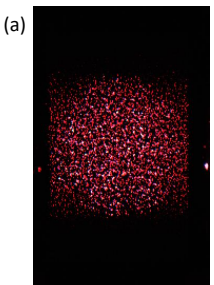

Sphere

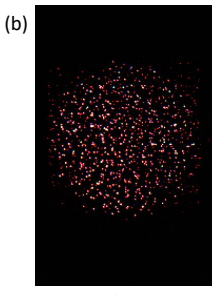

Sphere

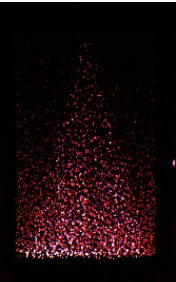

Cone

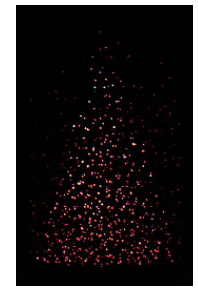

Cone

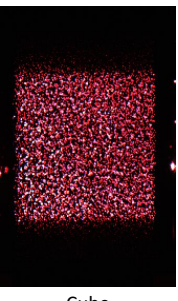

Cube

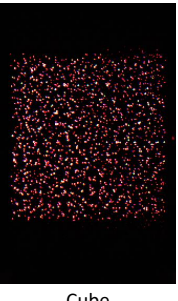

Cube
Fig. 14. Comparison between displayed images by (a)high-resolution display and (b)1-projector display.

ages' brightness and resolution by increasing the number of the projector to lighten voids was proposed. To verify the proposed method's effectiveness, two types of displays, one designed to improve especially the brightness and the other designed to improve especially, were developed, and subjective evaluation experiments were conducted. The display designed to improve especially the brightness resulted in the improvement of visibility, however, the display designed to improve the resolution dis not result in enhancing the visibility even though the resolution of the display was improved. It was likely that matching was not performed with enough accuracy, and that resulted in an increase of unnecessary luminescence. For future works, development of more accurate matching method and enhancement of visibility by reducing unnecessary luminescence should be considered.

\section{ACKNOWLEDGMENT}

This study was supported by JSPS KAKENHI Grant Number JP 26540044.

\section{REFERENCES}

[1] D. M. Hoffman, A. R. Girshick, K. Akeley, M. S. Banks, "Vergenceaccommodation conflicts hinder visual performance and cause visual fatigue," Journal of Vision, vol. 8, issue. 3, article 33, pp. 1-30, March 2008.

[2] S. Iwasawa, M. Kawakita, and N. Inoue, "REI: an automultiscopic projection display," Proceedings of Three Dimensional Systems and Applications, selected paper 1, pp. 1-4, June 2013.

[3] A. Jones, I. McDowall, H. Yamada, M. Bolas, P. Debevec, "Rendering for an interactive 360 light field display," ACM Transactions on Graphics, vol. 26, issue. 3, article 40, pp. 1-10, July 2007.

[4] H. Ishikawa, H. Saito, "Point cloud representation of 3D shape for laserplasma scanning 3D display," 34th Annual Conference of IEEE Industrial Electronics, pp.1913-1918, November 2008.

[5] S. K. Nayar, V. N. Anand, "3D display using passive optical scatterers," Computer, vol. 40, no. 7, pp. 54-63, July 2007.

[6] D. Ristau, Laser-Induced Damage in Optical Materials, CRC Press, December 2012. 